\title{
Biological rhythm disturbance in depression: temporal coherence of ultradian sleep EEG rhythms
}

\author{
R. ARMITAGE, ${ }^{1}$ R. F. HOFFMANN AND A. J. RUSH \\ From the Department of Psychiatry, University of Texas Southwestern Medical Center at Dallas, Texas, \\ $U S A$
}

\begin{abstract}
Background. Recent studies have suggested that major depressive disorders are associated with a breakdown in the organization of ultradian rhythm in sleep EEG. The present study used crossspectral analysis of sleep EEG to confirm this finding, in a larger-scale study, evaluating the influence of gender and age on ultradian rhythms in depression.

Methods. Temporal coherence of ultradian (80-120 min) rhythms in beta, theta and delta, recorded from central and parietal sites, were compared in 120 symptomatic, unmedicated, depressed outpatients and 59 healthy normal controls.

Results. Few macro-architectural differences were noted between patients and controls. However, interhemispheric beta and theta coherence and intrahemispheric coherence between beta and delta rhythms were significantly lower in depressed patients. Coherence measures were lowest in women with depression and highest in men in the control group, but were not strongly influenced by age. Over $65 \%$ of depressed patients were $\geqslant 2$ standard deviations below normal on at least one coherence measure, in sharp contrast to less than $10 \%$ of patients on macro-architectural variables.

Conclusions. It was concluded that dysregulation of ultradian rhythems characterizes the majority of depressed out-patients, primarily women, even when macro-architecture did not differentiate groups. The outcome of this study supports the view that the pathophysiology of depression is strongly influenced by gender. It was suggested that low temporal coherence in depression reflects a breakdown in the organization of sleep EEG rhythms within and between the two hemispheres.
\end{abstract}

\section{INTRODUCTION}

Rhythms are ubiquitous, present even in the most primitive of plant species (Mistlberger \& Rusak, 1989) and arise from self-organization of complex systems (Jantsch, 1980). Self-organization of arousal and activation in mammalian brain is reflected in circadian $(24 \mathrm{~h})$, ultradian (shorter than $24 \mathrm{~h}$ ) and infradian (longer than 24 h) rhythms in physiology, biochemistry and behaviour (Halberg, 1977).

The notion that major depressive disorders are associated with biological rhythm disturbances was introduced more than 40 years ago (Healy, 1987; Wirz-Justice, 1995). Abnormali-

${ }^{1}$ Address for correspondence: Dr Roseanne Armitage, Sleep Study Unit, University of Texas Southwestern Medical Center, 5323 Harry Hines Boulevard, Dallas, Texas, 75235-9070, USA. ties in rhythms could manifest in a shift in period length, a reduction in the amplitude or strength of the rhythm, or desynchronization and uncoupling of rhythms that usually covary, all of which have been described in depressed patients (Healy, 1987; Wirz-Justice, 1995). Two recent reviews, however, have concluded that there is no primary circadian rhythm disturbance in depression (Healy \& Waterhouse, 1995; WirzJustice, 1995). This has been most clearly demonstrated with temperature, the gold standard of circadian rhythms (Monk et al. 1994).

Nevertheless, a wealth of data indicate that neuroendocrine, hormonal and neurotransmitter regulation are compromised in depression (Janowsky et al. 1996). In addition, depression is associated with significant sleep disturbance, particularly with regard to the timing of REM sleep (Reynolds \& Kupfer, 1987). Taken to- 
gether, these findings suggest that depression may be better characterized by ultradian rather than circadian disturbance.

Depression has been associated with a short ( $<65 \mathrm{~min}$ ) latency to the first rapid eye movement (REM) sleep period, a shift in the distribution of REM sleep to the first half of the night, increased phasic activation during REM sleep and increased total REM time (Reynolds \& Kupfer, 1987; Kupfer et al. 1974; Armitage, 1995). Depressed patients also show a more rapid induction of REM sleep in response to a cholinergic challenge (Gillin et al. 1991; Riemann et al. 1991; Riemann \& Berger, 1992). Although REM sleep abnormalities are not specific to depression and have been reported in other psychiatric disorders (Benca et al. 1992), it is estimated that about $40-60 \%$ of out-patients show abnormal REM architecture (Armitage, 1995). Moreover, depression has also been associated with prolonged sleep onset, reduced slow-wave sleep, increased wakefulness and increased sleep fragmentation.

Quantitative EEG analyses during sleep, or so-called microarchitecture, have also revealed abnormalities in depressed patients. A number of studies have consistently reported loweramplitude delta activity or a reduction in the number of high-amplitude delta waves during non-rapid eye movement (NREM) sleep in depressed patients compared to healthy controls (Borbély et al. 1984; Kupfer et al. 1984, $1986 a$, b, 1990; Reynolds et al. 1990; Buysse et al. 1994; Armitage, 1995), although not all studies support this finding (Mendelson et al. 1987; Armitage et $a l .1992 b$ ). In addition, an abnormal time course in the accumulation and dissipation of delta has been described as a characteristic of some patients with major depressive disorder (MDD) primarily among men (Armitage et al. 1997). Although the precise nature of delta abnormalities is equivocal, most studies do indicate a disruption in the distribution of delta activity across the night in patients with MDD (Van den Hoofdakker \& Beersma 1986).

Sleep microarchitectural abnormalities in MDD are not just restricted to delta frequencies, but are also found in higher frequencies, most notably alpha (Borbély et al. 1984) and beta (Armitage et al. $1992 a, b, 1995$; Armitage, 1995) and in total EEG power (Borbély et al. 1984). Those with MDD show greater amplitude and incidence of high-frequency activity during sleep than that observed in healthy controls regardless of whether analysis are sleep-stage independent (Armitage et al. 1992b) or conducted within each sleep stage (Armitage et al. 1995). Of particular interest is the finding that women with MDD had the highest incidence of beta activity throughout sleep (regardless of sleep stage), yet also showed more delta than men with MDD in two independent studies (Armitage et al. 1995, 1997), suggesting that MDD is characterized by dysregulation of sleep EEG rhythms rather than simple hyperarousal.

Several researchers have suggested that both the sleep macro- and micro-architectural abnormalities in MDD may be accounted for by a single ultradian rhythm disturbance (Vogel et al. 1980; Beersma et al. 1984; Armitage, 1995; Armitage \& Hoffmann, 1997). In adults, primary ultradian rhythms have a 90 min periodicity. The $80-120$ min REM/NREM sleep cycle is perhaps the best documented ultradian 'rhythm' in adults. However, Webb has clearly demonstrated that changes in REM-NREM sleep stages do not meet criteria for a rhythm per se, as the interval between them shortens across the night and violates assumptions of stationarity (Webb, 1982). Nevertheless, the sleep cycle has been viewed as part of the ultradian basic restactivity cycle (BRAC) that persists throughout sleep and wakefulness and is coupled with rhythms in mood, alertness and cognitive performance (Kleitman, 1963; Broughton, 1975; Klein \& Armitage, 1979).

Our research group has had a long-standing interest in the 90 min ultradian rhythms in sleep micro-architecture that underlie REM-NREM cycles (Armitage, 1986, 1995; Armitage et al. 1992c; Armitage \& Hoffmann, 1997). Approximate 90 min rhythms in beta, theta and delta activity have been reported in healthy young adults in both sleep and wakefulness and are strongly correlated across sleep and wakefulness (Manseau \& Broughton, 1984; Okawa et al. 1984; Armitage, 1986; Armitage et al. 1989, 1992c).

We have also investigated ultradian rhythms in sleep EEG frequencies in depressed patients focusing on temporal coherence, a measure of the degree of synchrony or coupling between allnight EEG rhythms. An initial report of 18 depressed out-patients and age- and sex-matched 
controls indicated that temporal coherence between $90 \mathrm{~min}$ beta and delta rhythms was significantly lower in those with MDD (Armitage et al. 1992b). This study, however, included only the left central EEG electrode site, and thus constituted an evaluation of temporal coherence in intrahemispheric rhythms. A later report of an independent sample of 36 patients and controls confirmed lower temporal coherence between beta and delta rhythms in both left and right hemisphere recording sites and in interhemispheric beta rhythms in the MDD group (Armitage et al. 1993), consistent with the notion that the organization of ultradian rhythm is compromised in depression. Both of these studies, however, included relatively small sample sizes and did not take age and sex into account.

Micro-architectural abnormalities in MDD have also been shown to be strongly influenced by gender (Armitage, 1995; Armitage et al. 1995, 1997). Although sex differences have also been reported in healthy young adults (Dijk et al. 1989; Ehlers \& Kupfer, 1997), the magnitude of differences between depressed men and women appears to be substantially larger than those observed in controls (Armitage, 1995; Armitage et al. 1995, 1997; Armitage \& Hoffmann, 1997). By contrast, Reynolds et al. (1990) reported stronger main effects for age than for sex, although these effects were not evaluated with regard to discriminating patients from controls. In the latter context, sex effects may account for more variance than age (Armitage et al. 1997) and may also influence temporal coherence of sleep EEG rhythms.

The goal of the present study was to evaluate low temporal coherence in a large sample of outpatients with MDD $(N=120)$ and healthy controls $(N=59)$ with specific emphasis on sex and age effects.

\section{METHOD}

\section{Out-patients with MDD}

One hundred and twenty out-patients, 18-56 years of age (average $35 \cdot 5 \pm 10 \cdot 3$ years), who met criteria for non-psychotic, moderate to severe MDD and who had a minimum score of 18 on the 17-item Hamilton Rating Scale for Depression (Hamilton, 1960) were included in study. The sample included 45 men and 75 women. Diagnoses were made on the basis of the Structured Clinical Interview for DSM-IIIR (SCID) (Spitzer et al. 1986). Those patients with current general medical conditions, or current Axis I disorders in the categories of organic mental syndromes or disorders, bipolar disorders, schizophrenia, delusional or psychotic disorders were excluded. Patients with a history of psychoactive substance abuse within 12 months prior to study were also excluded as were those with other general medical conditions. All patients were medication-free for $\geqslant 2$ weeks ( $\geqslant 4$ weeks for MAOIs and $\geqslant 6$ weeks for SSRIs). Thus, the sample included those with moderate to severe depression who were symptomatic but unmedicated at the time of study. Demographic and clinical information on patients is shown in Table 1 .

\section{Healthy controls}

Fifty-nine healthy adults $18-55$ years of age (average $30.0 \pm 8.7$ years) who were screened for medical and psychiatric healthiness also participated in study. The SCID (Non-patient Version) confirmed the absence of past or present psychiatric illness in each participant. Further, no first-degree family members had a history of any psychiatric pathology, based on participant interview. The sample included 34 men and 25 women.

\section{Total sample}

Full medical and psychiatric examinations including lab tests and urine screens were conducted on all participants to ensure that inclusion and exclusion criteria were met. For both groups of participants, history of head trauma, shift work or failure to provide written informed consent excluded subjects from study. Any medication use except for non-steroidal anti-inflammatories was proscribed. In addition, women who were pregnant, lactating or sexually active and not using contraception were not enrolled. The study was approved by the Institutional Review Board and carried out in accordance with the tenets of the Declaration of Helsinki. The quantitative EEG data in this study has not been reported elsewhere, although the sleep macro-architecture on 80 of the depressed patients has been included as part of other reports (Armitage et al. 1994; Hendrickse et al. 1994; Trivedi et al. 1998). 
Table 1. Demographic and clinical information on out-patients with $M D D(\mathrm{~N}=120)$

\begin{tabular}{|c|c|c|c|c|c|c|}
\hline & \multicolumn{3}{|c|}{$\operatorname{Men}(N=45)$} & \multicolumn{3}{|c|}{ Women $(N=75)$} \\
\hline & Mean & (S.D.) & Range & Mean & (S.D.) & Range \\
\hline Age & $36 \cdot 7$ & $(8 \cdot 9)$ & $20-49$ & $34 \cdot 8$ & $(11 \cdot 0)$ & $18-56$ \\
\hline Education (in years) & $15 \cdot 1$ & $(1 \cdot 8)$ & $11-19$ & $13 \cdot 5$ & $(2 \cdot 3)$ & $9-24$ \\
\hline HRS-D* (17-item) & $20 \cdot 5$ & $(3 \cdot 4)$ & $18-30$ & $20 \cdot 8$ & $(5 \cdot 7)$ & $18-32$ \\
\hline IDS-C $\dagger(30$-item $)$ & $33 \cdot 2$ & $(7 \cdot 5)$ & $19-48$ & $36 \cdot 2$ & $(9 \cdot 7)$ & $19-53$ \\
\hline Age of Onset: (in years) & $27 \cdot 9$ & $(11 \cdot 6)$ & $10-49$ & $25 \cdot 1$ & $(11 \cdot 6)$ & $7-54$ \\
\hline \# Previous episodes & $2 \cdot 3$ & $(1 \cdot 7)$ & $1-7$ & $3 \cdot 1$ & $(2 \cdot 1)$ & $1-8$ \\
\hline Length of current episode (in months) & $35 \cdot 3$ & $(34 \cdot 8)$ & $3-128$ & $38 \cdot 5$ & $(42 \cdot 0)$ & $3-248$ \\
\hline \# First episode & \multicolumn{3}{|c|}{$N=22(49 \%)$} & \multicolumn{3}{|c|}{$N=33(44 \%)$} \\
\hline \# Recurrent & \multicolumn{3}{|c|}{$N=23(51 \%)$} & \multicolumn{3}{|c|}{$N=42(56 \%)$} \\
\hline
\end{tabular}

* Hamilton Rating Scale for Depression.

$\dagger$ Inventory of Depressive Symptomatology - Clinician Rated.

\$ Of first depressive episode.

\section{Procedures}

Each participant spent two consecutive nights in the University of Texas Southwestern Medical Center Sleep Study Unit, the first of which served as laboratory adaptation. All participants maintained regular bed- and rise-times for 5 days prior to study, verified by home diary. This habitual sleep schedule was also followed in the laboratory. Any subject with more than one half-hour deviation in sleep schedule during the 5 days was excluded from the study. All subjects slept 7-8 h in bed at home and in the laboratory. Subjects entered the laboratory for electrode application approximately one and one-half hours prior to scheduled bedtime.

The electrode montage included left (C3) and right (C4) central EEG, left (P3) and right (P4) parietal EEG, left and right electro-oculograms (EOG) recorded from the upper and lower canthi, and a bipolar, chin-cheek electromyogram (EMG). Night 1 also served as an additional screening for independent sleep disorders including apnea, bruxism and periodic limb movements and included leg leads, chest and abdomen respiration bands and a nasal-oral thermistor in the electrode montage. EEG electrodes were referenced to the ear lobes connected to a $10 \mathrm{k} \Omega$ resistor to minimize nonhomogeneous current flow and potential artefactual hemispheric asymmetries (Nunez, 1981), as is standard in our laboratory. EEG was transduced by GRASS ${ }^{\mathrm{TM}}$ P511 A/C amplifiers set at a sensitivity of $5(50 \mu \mathrm{V}, 0.5 \mathrm{~s}$ calibration), corresponding to a gain of 50000 . The half-amp low- and high-bandpass filters were set at $0 \cdot 3$ and $30 \mathrm{~Hz}$, respectively. A $60 \mathrm{~Hz}$ notch filter attenuated electrical noise.

Signals were digitized on-line at $250 \mathrm{~Hz}$ $(62.5 \mathrm{~Hz}$ for EOG and EMG) through a 16-bit MICROSTAR $^{\mathrm{TM}}$ analogue-to-digital (A/D) converter and displayed on a digital polygraph system designed and validated in-house. Raw digitized data were stored on a write-once-readmany (WORM) optical disk for off-line period amplitude analysis and the computation of temporal coherence.

Sleep records were scored according to standard criteria (Rechtschaffen \& Kales, 1968) by research personnel trained at better than $90 \%$ agreement on an epoch-by-epoch basis. All records were inspected visually and epochs containing movement, breathing or muscle artefact, or recording difficulties were excluded from analysis. An average of $5 \cdot 1 \pm 0 \cdot 8$ epochs were excluded resulting in the loss of $<3$ min of EEG data over the $7-8 \mathrm{~h}$ recording period.

\section{Signal processing}

There are several steps in computing temporal coherence of EEG rhythms. The first is to quantify sleep-stage independent, all-night EEG activity, usually accomplished through the use of period amplitude analysis (PAA) or power spectral analysis (PSA) describing incidence and amplitude or power, respectively, in each EEG frequency. The relative merits of the two techniques have been debated elsewhere (Ktonas \& Gosalia, 1981; Pigeau et al. 1981; Ktonas, 1987; Geering et al. 1993; Armitage \& Hoff- 
mann, 1997). Due to our interest in describing fast-frequency EEG activity, we chose PAA since it is biased toward fast-frequency events and has been particularly useful in capturing sex differences in patients with MDD (Armitage et al. 1995).

The complete details of the PAA algorithm have been described elsewhere (Hoffmann et al. 1979; Armitage et al. 1992 b). For the purposes of this report, delta $(0.5$ to $<4 \mathrm{~Hz})$, theta (4 to $<8 \mathrm{~Hz})$ and beta $(16-32 \mathrm{~Hz})$ incidence were quantified using a zero-cross analysis for delta and first-derivative analysis for theta and beta for each $30 \mathrm{~s}$ epoch of sleep. This decision to focus on these three frequency bands was driven by previous work demonstrating strong ultradian rhythms in these EEG bands in both healthy individuals (Manseau \& Broughton, 1984; Okawa et al. 1984; Armitage et al. 1992 c) and patients with MDD (Armitage et al. 1989, 1992 b, 1993; Armitage, 1995).

The second step in computing temporal coherence is to determine whether the changes in beta, theta and delta incidence are predictably recurrent throughout the night (i.e. show a ultradian rhythm with a fixed period) and to quantify the strength or amplitude of the rhythm, achieved through the use of bivariate crossspectral analysis (Gottman, 1981). Time series were constructed from beta, theta and delta incidence measures averaged across $1 \mathrm{~min}$ epochs. Cross-spectral power, coherence and period length were evaluated from 1-7 cycles in the entire $7-8 \mathrm{~h}$ recording period (i.e. maximum period of 420-480 min; minimum period of about $60 \mathrm{~min}$ ) separately for each subject. The period at which cross-spectral power was maximal was identified as the dominant rhythm in each pair of incidence measures. The figures below illustrate the information that is captured in interhemispheric beta coherence. In keeping with our previous work, we evaluate interhemispheric coherence between left and right beta rhythms, and between left and right theta rhythms. Intrahemispheric coherence is evaluated between beta and delta rhythms in the right and in the left hemispheres, and between theta and delta rhythms in the right and left hemispheres (Armitage et al. 1992b, 1993; Armitage, 1995; Armitage \& Hoffmann, 1997). Fig. 1 (top) illustrates beta incidence from left (C3) and right (C4) hemispheres recorded in a normal control (NC) woman. Note that beta incidence waxes and wanes periodically across the night. Left and right hemisphere beta incidence showed changes that were in-phase and resulted in high coherence at $96 \mathrm{~min}$. These left and right beta incidence values/epoch constitute the time series of 4801 -min epochs from which interhemispheric beta coherence was computed. The dominant rhythm was $96 \mathrm{~min}$ in each of left and right beta and between the two (cross-spectral power) and coherence at 96 min was $0 \cdot 99$, indicating high synchronization between left and right beta rhythms.

Fig. 1 (bottom) illustrates left and right beta incidence in an age- and gender-matched, unmedicated, symptomatic $\left(\mathrm{HRS}-\mathrm{D}_{17}=23\right)$ outpatient with MDD that resulted in very low beta coherence. Note that the phase relationship between left and right beta was inconsistent and changed erratically throughout the night, although both left and right beta showed 96 minperiod. The coherence between the two was dramatically lower $(0.43)$ than that obtained from the NC above. The cross-spectral power, however, was not significantly lower than the $\mathrm{NC}$ and shows the same period, indicating that it is not dampened or phase-shifted ultradian rhythmicity, but low coherence, i.e. desynchronized left and right beta rhythms that characterizes this patient and others with MDD. Intrahemispheric coherence measures are derived in a similar fashion.

\section{Macro-architectural variables}

Although the key focus of this paper was on temporal coherence of ultradian rhythms, sleepstage variables were also included for comparative and interpretative purposes. These included: total time in bed (TIB), total sleep period (TSP), sleep latency and REM latency, expressed in minutes. Time in bed was defined as the minutes of the total recording period. The total sleep period was defined as the minutes from persistent sleep onset to morning awakening. The minutes and percentage of sleep stages were expressed relative to total sleep period. Sleep latency was defined as the time in minutes to the first 10 min segment of any sleep stage, with not more than $2 \mathrm{~min}$ of intervening wakefulness, i.e. latency to persistent sleep (Armitage et al. 1994). REM latency was defined as the minutes from sleep onset to the first epoch 

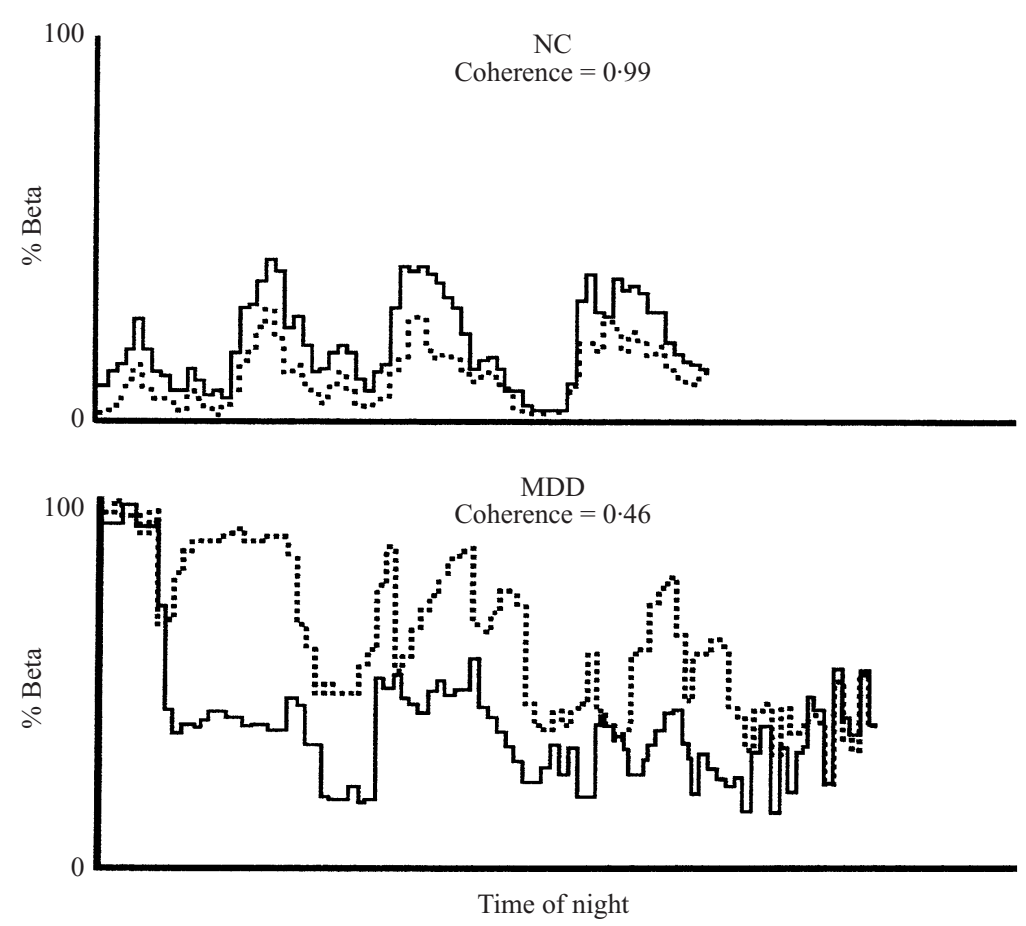

Fig. 1. All-night beta incidence from left $(\mathrm{C} 3,-\longrightarrow)$ and right $(\mathrm{C} 4,----)$ recording sites from period amplitude analysis in a $22-$ year old healthy normal control (NC) woman (top) and from an age- and gender-matched, unmedicated out-patient with majo depressive disorder (MDD).

of REM sleep with no minimum duration criterion. Sleep efficiency (TSP/TIB), the number of arousals $(>0.5 \mathrm{~min})$ and average REM density were also computed. REM density was computed on a four-point scale and averaged for each minute of REM sleep.

\section{Statistical procedures}

Following cross-spectral analysis, the coherence, periodicity and power values were entered into a dataset along with age, and coded for group (MDD v. NC) and sex, the between-group variables. An age cutpoint of above or below 30 years was used, following Ehlers \& Kupfer (1997) who demonstrated that age- and genderrelated changes in delta activity were more apparent in healthy adults after the age of 30 . For interhemispheric measures, EEG frequency band was treated as a repeated hemisphere. For the intrahemispheric measures, EEG frequency band and hemisphere were treated as nested, two-level, repeated measures. Multivariate analyses of variance (MANOVAs) were used to evaluate between-group differences. Separate
$2 \times 2 \times 2$ MANOVAs were computed on interand intra-hemispheric measures derived from central and parietal recording sites. The sex by group by age interactions were tested first, followed by two-way interactions and main effects, if a significant overall MANOVA effect was found, using an experiment-wise $\alpha=0.05$. For additional protection against Type I errors, the conservative Wilks-Lambda $F$ ratio was used to determine significance of MANOVA effects. Least-squares multiple comparison tested individual mean differences for significant effects. Two MANOVAs were computed on the sleep macro-architectural variables following the same statistical rules as outlined above. The percentage of sleep states (1,2, SW and REM) were treated as a four-level repeated measure. The percentage awake was excluded from the repeated-measures MANOVA to avoid multicolinearity and an over determined matrix (see Pedhazur, 1982, p. 232). The percentage awake was included with the other six macro-architectural variables in a between-group $2 \times 2 \times 2$ MANOVA. Pearson's product moment corre- 
lations evaluated the relationship between macro- and micro-architecture. All primary statistical analyses were based on Night 2 data.

\section{RESULTS}

\section{Macro-architecture}

The means and standard deviations of the macroarchitectural variables are shown in Table 2. For the most part, men with MDD showed the most disrupted sleep, particularly in those over 30 years of age. By contrast, sleep macroarchitecture in the women with MDD was relatively 'normal', with the exception of prolonged sleep latency in older depressed women. Only the percentage sleep stage measures differed between groups (see below).

The between-groups MANOVA revealed a significant overall group by sex by age effect $(F=1 \cdot 5, \mathrm{df}=56,889, P<0.02)$, a group by sex interaction $(F=2.3, \mathrm{df}=24,488, P<0.0007)$ and main effects for group $(F=2 \cdot 2, \mathrm{df}=8,168$, $P<0.03)$ and sex $(F=2.7, \mathrm{df}=8,168, P<$ 0.009). However, univariate analyses revealed that only one of the seven variables entered into the MANOVA model showed significant effects, namely percentage awake. A significant three-way interaction $(F=5 \cdot 3, \quad \mathrm{df}=4,174$, $P<0.0005)$, a group by sex effect $(F=7 \cdot 8$, $\mathrm{df}=3,175, P<0.0001)$ and main effects for group $(F=3.9, \mathrm{df}=1,175, P<0.05)$ and sex $(F=15 \cdot 7, \quad$ df $=1,175, P<0.0001)$ were ob- tained from ANOVA. Multiple comparisons indicated that men with MDD, regardless of age, had more percentage awake than all other groups ( $P$ range: 0.002-0.0001). None of the other individual means differed significantly.

With regard to the other percentage Stage measures, repeated-measures MANOVA revealed an overall significant sleep stage by group by sex by age interactions $(F=3 \cdot 6, \mathrm{df}=21,486$, $P<0.0001)$. Univariate ANOVAs indicated group by sex by age interactions for percentage Stage $1(F=5 \cdot 2, \mathrm{df}=7,17, P<0.001)$, percentage SW $(F=6 \cdot 8$, df $=7,171, P<0.0001)$ and percentage Stage $2(F=2 \cdot 22, \mathrm{df}=7,171$, $P<0.04)$. The percentage Stage 1 also showed group by sex $(F=8 \cdot 1, \mathrm{df}=3,175, P<0 \cdot 001)$, and group by age interactions $(F=3 \cdot 2$, $\mathrm{df}=3,175, P<0 \cdot 02$ ), along with main effects for $\operatorname{sex}(F=16 \cdot 4, \mathrm{df}=1,175, P<0 \cdot 0001)$ and age $(F=5 \cdot 8, \mathrm{df}=1,175, P<0.02)$.

Least-squares multiple comparisons confirmed that percentage Stage 1 sleep was significantly higher in MDD men over 30 than all other groups ( $P$ range: $0 \cdot 005-0 \cdot 0001)$. No other individual mean differences were significant for this measure. The percentage SW also showed group by sex $(F=5.8, \mathrm{df}=8,175, P<0.0008)$ and group by age interactions $(F=11.9, \mathrm{df}=$ $3,175, P<0.0001)$ along with main effects for group $(F=5 \cdot 0, \quad \mathrm{df}=1,175, \quad P<0 \cdot 03), \quad$ sex $(F=9 \cdot 5, \mathrm{df}=1,175, P<0 \cdot 002)$ and age $(F=$ $28 \cdot 4$, df $=1,175, P<0 \cdot 0001)$. Multiple com-

Table 2. Means ( \pm standard deviations) of key sleep-stage variables by group, sex and age

\begin{tabular}{|c|c|c|c|c|c|c|c|c|}
\hline \multirow[b]{2}{*}{$\begin{array}{l}\text { Sleep stage } \\
\text { variable }\end{array}$} & \multicolumn{4}{|c|}{ Under 30} & \multicolumn{4}{|c|}{ Over 30} \\
\hline & $\begin{array}{c}\mathrm{NC} \\
\text { Men } \\
(N=23)\end{array}$ & $\begin{array}{c}\mathrm{NC} \\
\text { Women } \\
(N=12)\end{array}$ & $\begin{array}{c}\text { MDD } \\
\text { Men } \\
(N=7)\end{array}$ & $\begin{array}{c}\text { MDD } \\
\text { Women } \\
(N=27)\end{array}$ & $\begin{array}{c}\mathrm{NC} \\
\text { Men } \\
(N=11)\end{array}$ & $\begin{array}{c}\mathrm{NC} \\
\text { Women } \\
(N=13)\end{array}$ & $\begin{array}{c}\text { MDD } \\
\text { Men } \\
(N=38)\end{array}$ & $\begin{array}{c}\text { MDD } \\
\text { Women } \\
(N=48)\end{array}$ \\
\hline Time in bed & $427 \cdot 9(43 \cdot 5)$ & $418 \cdot 8(40 \cdot 9)$ & $455 \cdot 7(75 \cdot 3)$ & $448 \cdot 8(53 \cdot 9)$ & $412 \cdot 6(45 \cdot 5)$ & $444 \cdot 8(48 \cdot 9)$ & $432 \cdot 2(44 \cdot 6)$ & $434 \cdot 9(56 \cdot 7)$ \\
\hline Total sleep period & $409 \cdot 0(46 \cdot 8)$ & $406 \cdot 4(37 \cdot 0)$ & $433 \cdot 8(83 \cdot 1)$ & $430 \cdot 4(52 \cdot 9)$ & $404 \cdot 7(45 \cdot 8)$ & $430 \cdot 5(43 \cdot 8)$ & $417 \cdot 0(46 \cdot 7)$ & $413 \cdot 3(61 \cdot 3)$ \\
\hline Sleep efficiency & $95 \cdot 8(7 \cdot 6)$ & $97 \cdot 2(3 \cdot 0)$ & $94 \cdot 9(5 \cdot 7)$ & $95 \cdot 9(2.9)$ & $98.0(1.9)$ & $97 \cdot 0(3 \cdot 3)$ & $96 \cdot 4(3 \cdot 0)$ & $94.9(5 \cdot 9)$ \\
\hline Sleep latency & $19 \cdot 5(15 \cdot 7)$ & $9 \cdot 8(9 \cdot 7)$ & $10 \cdot 1(8 \cdot 2)$ & $17 \cdot 4(11 \cdot 7)$ & $5 \cdot 7(4 \cdot 7)$ & $11.8(8 \cdot 9)$ & $13 \cdot 2(10 \cdot 2)$ & $18 \cdot 8(24 \cdot 3)$ \\
\hline REM latency & $79 \cdot 1(30 \cdot 1)$ & $67 \cdot 6(10 \cdot 7)$ & $76 \cdot 8(17 \cdot 3)$ & $74 \cdot 1(23 \cdot 7)$ & $71 \cdot 4(59 \cdot 3)$ & $67 \cdot 2(13 \cdot 6)$ & $74 \cdot 6(24 \cdot 6)$ & $80 \cdot 7(37 \cdot 8)$ \\
\hline Stage $(\%) 1 * \dagger$ & $14 \cdot 0(5 \cdot 7)$ & $12 \cdot 8(4 \cdot 8)$ & $13 \cdot 7(5 \cdot 0)$ & $14 \cdot 7(6 \cdot 2)$ & $17 \cdot 4(7 \cdot 5)$ & $15 \cdot 3(8 \cdot 8)$ & $21 \cdot 1(6 \cdot 9)$ & $14 \cdot 3(5 \cdot 3)$ \\
\hline Stage $(\%) 2 \dagger$ & $53 \cdot 3(7 \cdot 9)$ & $52 \cdot 3(4 \cdot 3)$ & $53 \cdot 6(9 \cdot 7)$ & $53 \cdot 5(8 \cdot 9)$ & $57 \cdot 0(7 \cdot 4)$ & $55 \cdot 5(10 \cdot 4)$ & $50 \cdot 5(8 \cdot 0)$ & $52 \cdot 0(8 \cdot 5)$ \\
\hline $\begin{array}{l}\text { Slow-wave }(\%)^{*} \uparrow \\
(\text { Stage } 3+4)\end{array}$ & $9 \cdot 3(7 \cdot 2)$ & $10 \cdot 4(7 \cdot 4)$ & $5 \cdot 3(5 \cdot 7)$ & $8 \cdot 7(6 \cdot 4)$ & $2 \cdot 1(2 \cdot 1)$ & $3 \cdot 5(3 \cdot 2)$ & $1 \cdot 4(2 \cdot 1)$ & $5 \cdot 1(7 \cdot 9)$ \\
\hline REM (\%) & $18 \cdot 1(4 \cdot 7)$ & $21 \cdot 2(3 \cdot 0)$ & $17 \cdot 5(5 \cdot 6)$ & $19 \cdot 1(5 \cdot 0)$ & $17 \cdot 6(4 \cdot 1)$ & $20 \cdot 3(3 \cdot 8)$ & $18 \cdot 4(5 \cdot 0)$ & $18 \cdot 0(5 \cdot 5)$ \\
\hline Awake $(\%) * \dagger$ & $5 \cdot 3(3 \cdot 0)$ & $3 \cdot 3(1 \cdot 0)$ & $9 \cdot 8(13 \cdot 3)$ & $4 \cdot 0(2 \cdot 4)$ & $5 \cdot 9(5 \cdot 3)$ & $5 \cdot 4(4 \cdot 4)$ & $8 \cdot 6(4 \cdot 9)$ & $5 \cdot 6(0 \cdot 7)$ \\
\hline REM density & $2 \cdot 2(0 \cdot 6)$ & $2 \cdot 3(0 \cdot 7)$ & $2 \cdot 4(0.9)$ & $2 \cdot 5(0 \cdot 6)$ & $2 \cdot 1(0 \cdot 5)$ & $2 \cdot 3(0 \cdot 7)$ & $2 \cdot 4(0 \cdot 7)$ & $2 \cdot 2(0 \cdot 7)$ \\
\hline
\end{tabular}

* Significant group main effect from ANOVA $P<0 \cdot 05$.

$\uparrow$ Significant group interaction from ANOVA $P<0.05$.

Percentage of sleep stages expressed relative to total sleep period (from sleep onset to morning awakening). 
Table 3. Means $( \pm$ standard deviations) of coherence variables derived from central EEG leads on night 2 by group and sex, collapsed across age

\begin{tabular}{|c|c|c|c|c|}
\hline Coherence variable & $\begin{array}{l}\text { NC Men } \\
(N=34)\end{array}$ & $\begin{array}{l}\text { NC Women } \\
\quad(N=25)\end{array}$ & $\begin{array}{l}\text { MDD Men } \\
(N=45)\end{array}$ & $\begin{array}{l}\text { MDD Women } \\
\quad(N=75)\end{array}$ \\
\hline \multicolumn{5}{|l|}{ Interhemispheric } \\
\hline Beta $(\mathrm{BCOH})^{*}$ & $0.95(0.06)$ & $0.94(0.06)$ & $0 \cdot 89(0 \cdot 11)$ & $0 \cdot 86(0 \cdot 10)$ \\
\hline Theta $(\mathrm{TCOH})^{*}$ & $0.96(0.04)$ & $0.96(0.13)$ & $0.86(0.14)$ & $0.85(0 \cdot 18)$ \\
\hline \multicolumn{5}{|l|}{ Intrahemispheric } \\
\hline Right beta delta $(\mathrm{BDRCOH}) * \dagger$ & $0.82(0.14)$ & $0.88(0.20)$ & $0.76(0.17)$ & $0 \cdot 65(0 \cdot 22)$ \\
\hline Left beta delta (BDLCOH)* $*$ & $0.83(0.14)$ & $0.86(0.13)$ & $0.73(0.21)$ & $0.69(0.23)$ \\
\hline Right theta delta (TDRCOH)* & $0.84(0.13)$ & $0.90(0.17)$ & $0.75(0.20)$ & $0.73(0 \cdot 19)$ \\
\hline Left theta delta (TDLCOH) & $0.92(0.06)$ & $0.87(0 \cdot 16)$ & $0.80(0.17)$ & $0.74(0.23)$ \\
\hline
\end{tabular}

* Significant group main effect from ANOVA $P<0.05$.

$\dagger$ Significant group interaction from ANOVA $P<0.05$.

parisons indicated that normal control women under 30 had more percentage SW than all other groups except $\mathrm{NC}$ men and MDD women $<30$ ( $P$ range: 0.007-0.0001). Men with MDD over 30 had less percentage SW than all other groups ( $P$ range: 0.006-0.0001) except $\mathrm{NC}$ men and women $>30$ years of age. Interestingly, MDD women were the only ones who did not shown a significant decline in percentage SW with age.

The percentage Stage 2 also showed a significant group by sex interaction $(F=3 \cdot 0, \mathrm{df}=$ $3,175, P<0.05)$ but not a group by age effect $(F<1)$. A sex main effect was also evident $(F=5 \cdot 2, \mathrm{df}=1,175, P<0 \cdot 02)$. Multiple comparisons indicated that MDD men over 30 had less percentage Stage 2 than NC men over 30 and MDD women in both age groups ( $P$ range: $0 \cdot 03-0 \cdot 0004)$. None of the other individual means differed significantly on this measure.

In summary, only the percentage sleep stage variables differentiated patients with MDD from controls due primarily to increased percentage Stage 1 and percentage awake and decreased percentage Stage 2 and percentage SW in men with MDD over 30.

\section{Micro-architecture \\ Periodicity and amplitude}

The average periodicity of ultradian rhythms derived from central electrode sites was 118.2 $\pm 32 \cdot 1$ (range $=80-130$ ) $\mathrm{min}$ in the $\mathrm{NC}$ group and $125.8 \pm 91 \cdot 3$ (range $=68-180)$ min in the MDD group. Shorter periodicity was found for interhemispheric beta coherence and theta activity with longer rhythms in intrahemispheric measures in both groups of subjects. In general, periodicity tended to be shorter in women than in men in the MDD groups, contributing to the relatively large variance in the MDD group. However, MANOVA did not reveal significant main effects or interactions on any of the periodicity measures $(F<1)$. Periodicity of ultradian rhythms derived from parietal electrode sites also did not differ significantly by group, sex or age $(F=<1)$. The average periodicity was $119 \cdot 3 \pm 29 \cdot 6$ in the NC group and $128 \cdot 4 \pm 62 \cdot 8$ in the MDD group. Thus, the periodicity measures did not show differences between groups, providing little evidence of phase-shifted ultradian rhythms in those with depression.

The average strength or amplitude of the ultradian EEG rhythms, as reflected in crossspectral power at the dominant period, was $1 \cdot 9 \pm 0 \cdot 8 \times 10^{3}$ (range: $\left.0 \cdot 8-2 \cdot 8 \times 10^{3}\right)$ in depressed patients and $1.8 \pm 0 \cdot 6 \times 10^{3}$ (range: $1 \cdot 0-3 \cdot 1 \times 10^{3}$ ) in healthy controls. No systematic betweengroup differences were noted, supported by $F<1$ for group and sex main effects and for the interactions. Although age effects were not significant, there was some evidence that the strength of ultradian rhythms declined with age, independent of depression $(F=2 \cdot 0, \mathrm{df}=1,175$, $P<0 \cdot 15)$.

\section{Interhemispheric coherence}

The means and standard deviations of the coherence measures derived from central sites are shown in Table 3 by group and sex. Coherence was lower in the group with MDD compared to controls, especially in women.

Repeated-measures MANOVA of coherence data derived from central sites revealed no significant group by sex by age or group by sex 

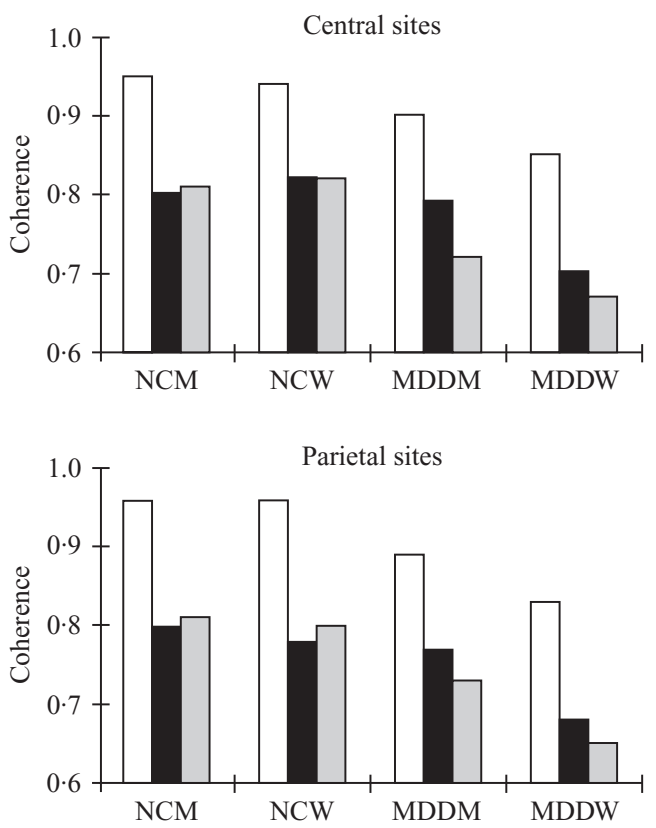

FIG. 2. Mean interhemispheric beta coherence (BCOH, $\square$ ), intrahemispheric beta-delta coherence in the right (BDRCOH, $\mathbf{\square})$ and left (BDLCOH, 圆) hemispheres in central (top) and parietal (bottom) recording sites for normal control men (NCM), normal control women (NCW), depressed men (MDDM) and depressed women (MDDW) collapsed across age and night in study.

interactions for interhemispheric coherence $(F<1)$. However, overall group and sex main effects were evident $(F=4 \cdot 2,3 \cdot 9$; respectively, $\mathrm{df}=1,175, P<0 \cdot 05)$. Age showed no significant effect on interhemispheric coherence $(F=<1)$. Univariate analyses confirmed lower beta coherence in the depressed group than in controls $(F=6 \cdot 2$, df $=1,176, P<0 \cdot 03)$. Interhemispheric theta coherence showed only a group main effect from ANOVA $(F=3 \cdot 9$, df $=1,176, \quad P<0.05)$. The sex main effect approached significance for theta coherence $(F=2.9$, df $=1,176, P<0.08)$ but not beta. Thus, interhemispheric beta and theta coherence were significantly lower in depressed patients than controls, and were influenced by sex but not age.

\section{Intrahemispheric coherence}

Intrahemispheric coherence between beta and delta showed a significant group by sex by age interaction from MANOVA $(F=3 \cdot 85$, $\mathrm{df}=7,171, P<0 \cdot 05)$. Univariate analyses confirmed three-way interactions for coherence between beta and delta in both the left and right hemispheres $(F=2 \cdot 8 ; 3 \cdot 1$, respectively, $\mathrm{df}=$ 7, 171, $P<0 \cdot 01)$. Coherence between beta and delta also showed a marginal group by sex effect $(F=2 \cdot 6$, df $=3,175, P<0 \cdot 09)$, a group by age interaction $(F=5 \cdot 1$, df $=3,175, P<0.009)$ and group main effects in both the left and right hemispheres $(F=14.1 ; 7 \cdot 2$ respectively, $\mathrm{df}=$ $1,175, P<0.008)$. Overall, the depressed group had significantly lower coherence between beta and delta rhythms than controls. Multiple comparisons confirmed lowest coherence in women with MDD regardless of age ( $P$ range: 0.05-0.007). Older, but not younger, men with MDD showed lower coherence than women in the control group, thus accounting for the threeway interaction. However, the effect size was largest for between-group differences.

Intrahemispheric coherence between theta and delta rhythms showed a group main effect $(F=4 \cdot 5$, df $=1,175, P<0.04)$ but no significant interactions. Univariate analyses indicated that depressed patients had lower coherence than controls but only between theta and delta rhythms in the right hemisphere $(F=4 \cdot 1$, $\mathrm{df}=1,175, P<0 \cdot 05)$. Although these coherence measures were also consistently lowest in women with MDD, sex did not account for a substantial portion of the overall variance.

The results from the coherence analyses were in contrast to the macro-architectural findings, which showed worse sleep in men with MDD, whereas lowest values for coherence were evident in women with MDD. Moreover, lower coherence among women with MDD was evident in both those older and younger than 30 years of age. Group main effects were stronger than interactions with sex and age, again in contrast to macro-architectural variables. In fact, age main effects were not evident in any coherence measures.

The analyses of coherence data derived from parietal sites showed very similar results, with group main effects and group by sex interactions on interhemispheric beta coherence and coherence between beta and delta in both hemispheres $(P<0.05)$. Those with depression had lower coherence than normal controls, with lowest coherence in depressed women.

To illustrate the comparability of betweengroup differences in coherence from central and parietal sites, Figure 2 presents the mean 
differences in interhemispheric beta and intrahemispheric coherence between beta and delta between groups. Note the pattern within and between groups is virtually identical in both electrode sites.

\section{Sensitivity in discriminating $M D D$ from $N C$}

Also of interest was the degree of sensitivity of macro- and micro-architectural measures in differentiating those with MDD from NCs. We first determined the number of subjects who were $\geqslant 2$ s.D.s outside $\mathrm{NC}$ means on each of the key measures. For sleep latency, percentage Stage 1 and percentage Awake, the criterion was the age-adjusted mean of the NC group plus 2 S.D.s, whereas the mean minus 2 s.D.s was used for REM latency, sleep efficiency, percentage SW sleep and all coherence measures. None of the 120 MDD subjects fell outside criteria on all variables, whereas less than $10 \%$ were identified as abnormal on at least one variable. For the individual variables, 5/120 (4\%) MDD subjects fell outside criterion on REM latency and on sleep latency. The percentage Stage 1 identified six subjects $(5 \%)$ as abnormal, five of whom were men. Only $3 / 59(5 \cdot 1 \%)$ NCs fell outside criterion. Thus, the distribution of macroarchitectural variables was relatively normal in both groups of subjects.

The coherence measures identified a larger number of patients with MDD as $\geqslant 2$ s.D.s outside age-adjusted normal values. Using the criterion of $\geqslant 2$ s.D.s on at least one coherence measure, $84 / 120(67 \%)$ were identified as abnormal. Twenty-five per cent $(30 / 120)$ were outside criteria on all six coherence measures, the majority of whom were women. With regard to specific measures, interhemispheric coherence identified $32-50 \%$ of patients as abnormal whereas intrahemispheric defined $30-57 \%$ as at least 2 s.D.s below the normal control mean. About $5 \%$ of normals fell outside the mean range. In addition to identifying a high number of low values, the distribution of coherence values was strongly bimodal in the depressed group, again in sharp contrast to macroarchitectural measures.

\section{Reliability of coherence measures}

The reliability of temporal coherence is central to the utility of the measures in differentiating depressed patients from healthy controls. To address this issue, MANOVA contrasted coherence values using night in study and electrode site as repeated measures. We reasoned that since laboratory adaptation effects are generally quite dramatic, that a failure to obtain a significant change in coherence from the first to second night in study would provide strong evidence for the reliability of the measures. The MANOVA provided $F$ ratios $<1$ for both electrode site and night. As a result, data are not shown. Temporal coherence was remarkably stable across nights and recording sites.

\section{Relationship between macro- and micro- architecture}

As highlighted above, the macro- and microarchitectural measures present a different view of sleep abnormalities in depression, as seen by contrasting Tables 2 and 3. To evaluate further the relationship between macro-architecture and coherence, correlations were computed between the two sets of measures. Since between-group differences were evident in both sets, separate Pearson's correlations were computed by group and sex.

Overall, macro-architectural and coherence measures were largely unrelated. Women in the NC group showed a significant correlation between interhemispheric beta coherence and REM latency $(r=-0.45 ; P=0.04)$, indicating that as REM latency increased, beta coherence decreased. Men in the NC group also showed a negative correlation between these variables which approached significance $(r=-0.36 ; P=$ $0 \cdot 06)$. No other correlations were significant in $\mathrm{NC}$ men and women. Interhemispheric beta coherence also correlated with percentage REM $(r=0.24 ; P=0.03)$ but only in women with MDD. In both men and women with MDD no other correlations were significant. Thus, the macro-architecture and coherence measures were largely independent, indicating that they reflect different aspects of brain organization during sleep.

\section{DISCUSSION}

The macro-architectural findings reported here are at least in partial agreement with previous work (Reynolds \& Kupfer, 1987; Kupfer et al. $1989 a, b$; Reynolds et al. 1990; Buysse et al. 1994), even though REM latency did not 
discriminate groups. The findings support the view that a cluster of variables, rather than one individual measure, best characterizes sleep macro-architecture in MDD (Benca et al. 1992). Moreover, age effects were stronger than group differences and were larger than sex effects, although significant interactions were also evident. Although depressed men over 30 years of age tended to have more disturbed sleep, only percentage awake and Stages 1 and 2 differed significantly from $\mathrm{NC}$ men in the same age group. Under age 30, only percentage awake differentiated depressed men from controls. By contrast, none of the macro-architectural measures distinguished depressed women from control women, regardless of age. There are several factors that may contribute to our failure to find macro-architectural differences. First, the length of the current episode of depression in this sample was more than 30 months. Recent work has shown that sleep abnormalities are more dramatic earlier in the episode (Dew et al. 1996) and thus our failure to find strong betweengroup differences may be due to the long length of the current episode. Secondly, our sample was relatively young, with the majority of patients under 40 years of age. Reynolds and colleagues have clearly demonstrated that sleep disturbances are even more dramatic in older patients (Reynolds et al. 1990). Indeed, between-group differences were stronger in men over 30 years of age. Out-patient status and regularized sleep schedules prior to the lab visit also could have minimized the degree of sleep disturbance in the depressed patients. Nevertheless, the small between-group differences in macro-architecture make the temporal coherence findings even more striking.

To summarize micro-architectural findings, period length and amplitude of ultradian rhythms in beta and theta did not differ significantly between depressed patients and controls. Thus, there was no evidence of phaseshifted or dampened rhythms in the those with depression. Temporal coherence of ultradian rhythms did discriminate between groups, suggesting that desynchronization and uncoupling of sleep EEG rhythms is characteristic of depressed patients. Interhemispheric beta coherence and intrahemispheric beta-delta coherence was significantly lower in those with MDD compared to controls. Inter- and intra- hemispheric theta coherence measures also tended to be lower in those with MDD. These differences were evident in both central and parietal recording sites and did not appear to show laboratory adaptation or 'first night' effects. Note, however, that temporal coherence was strongly influenced by gender in those with depression.

Using the criterion of 2 s.D.s below normal control means, coherence measures identified $30-70 \%$ of those with MDD as abnormal, in comparison to $0-5 \%$ on macro-architectural measures. A full $25 \%$ of patients were $\geqslant 2$ s.D.s below normal on all six coherence measures. In addition, the distribution of macro-architectural variables was relatively normal in the depressed group, whereas coherence variables showed a marked bimodal distribution. Thus, it was not just a few outliers with extreme values that contributed to lower mean coherence, but a large group of individuals, most of whom were depressed women.

These temporal coherence findings provide strong support for the notion that a primary ultradian rhythm disturbance characterizes depression, as suggested by Beersma et al. (1984) and Vogel et al. (1980), although it is the uncoupling and dysregulation of ultradian EEG rhythms rather than a phase-advance or dampened amplitude that appears to be characteristic of depression. However, the caveat must be added that ultradian rhythm disturbances are more characteristic of depressed women than of men. Our additional work indicates that men with depression are more likely to show reductions in slow-wave activity in NREM sleep and an abnormal time course in the accumulation and dissipation of slow-wave activity (Armitage et al. 1997, 1999). These findings are more consistent with homeostatic and sleep regulatory impairment in depressed men in keeping with the slow-wave sleep deficiency hypothesis of Borbély \& Wirz-Justice (1982). Thus, it appears that depressed women are more likely to show ultradian rhythm dysregulation and depressed men show slow-wave sleep regulatory impairment.

The finding of lower coherence in women with MDD is also consistent with the well-known increased prevalence of depression among women. It is estimated that after puberty, women are at twice the risk for the development of 
depression than are men (Halbreich \& Lumley, 1993; Heller, 1993; Kessler et al. 1993, 1994). The observation of more dramatic micro-architectural abnormalities in women with MDD and greater macro-architectural disturbances in men with MDD also adds substantial support to the view that the pathophysiology of depression differs for men and women (Halbreich \& Lumley, 1993; Heller, 1993; Armitage, 1995; Armitage et al. 1995, 1997; Armitage \& Hoffmann, 1997).

Since the strongest effects were evident in interhemispheric beta coherence and coherence between beta and delta rhythms in both the left and right hemispheres, one might conclude that primary ultradian disturbance is in fastfrequency rhythms, reflecting simple hyperarousal in those with depression. However, as previously shown by Kupfer et al. (1986a), Armitage (1995), Armitage et al. (1995), sleep fragmentation and intermittent wakefulness do not account for micro-architectural abnormalities. On the contrary, including only epochs of unambiguous SW sleep, elevated beta activity was still evident in the MDD group compared to controls, and highest in women with MDD (Armitage et al. 1997). Depressed women showed concomitant elevations in beta and delta activity, more consistent with global EEGfrequency dysregulation as a characteristic of MDD rather than hyperarousal. Moreover, the macro-architectural data reported here indicate that men with MDD had more Stage 1 sleep and wakefulness during the night, but it was the women with MDD who had lowest coherence in beta and delta rhythms. Thus, it is not simply elevated beta that produces low coherence.

We offer an alternative interpretation, namely that low inter- and intra-hemispheric beta coherence reflects a breakdown in the fundamental organization of arousal and activation during sleep in depressed women. Such an interpretation accounts for both the continued presence of delta activity and SW sleep coupled with increased beta activity reported in women with MDD (Armitage, 1995; Armitage et al. 1995, 1997) and the reduction and altered time course of delta in men with MDD (Armitage et al. 1997). Since coherence, but not periodicity or amplitude, of ultradian rhythms was found to differ from controls, it seems likely that the inconsistent phase relationship and the loss of coordination in left and right beta activity and between fast- and slow-frequency rhythms is responsible for low coherence in depressed women.

We further speculate that low coherence in ultradian EEG rhythms during sleep reflects a compromised and disorganized basic restactivity cycle that persists throughout sleep and wakefulness (Kleitman, 1963). This interpretation suggests that those with MDD should also show low coherence of ultradian rhythms in waking EEG activity. To date, we are unaware of a daytime ultradian EEG study in MDD, although there is ample evidence that those with depression do show abnormalities in EEG frequencies recorded during 20-60 min of wakefulness (Pollock \& Schneider, 1989). Furthermore, recent data indicate that rest-activity cycles, as measured by actigraphy, are indeed compromised in both adults and children with MDD with an overall disorganization that is more ultradian than circadian in nature (Teicher et al. 1993, 1997).

The outcome of this study indicates that temporal coherence of ultradian rhythms in sleep EEG discriminates depressed patients, primarily women, from normal controls even when macro-architectural measures do not. The future direction of our research will evaluate whether low coherence is also a characteristic of other psychiatric illnesses or is unique to depression, and whether these measures are state or trait features of depression and the clinical correlates of low coherence.

We wish to thank Dr Kenneth Altshuler, Chair of the Department of Psychiatry for administrative support, Doris Benson for secretarial support and the technical staff of the Sleep Study Unit, Darwynn Cole, supervisor. This research was supported in part by NIMH Grant no. 46886 (Dr Roseanne Armitage), NIMH Grant no. 41115 (Dr A. John Rush) and the Sarah M. and Charles E. Seay Center for Brain and Applied Research in Psychiatric Illness.

\section{REFERENCES}

Armitage, R. (1986). Ultradian Rhythms in EEG and Performance: An Assessment of Individual Differences in the Basic Rest-Activity Cycle. Ph.D. thesis. Carleton University: Ottawa.

Armitage, R. (1995). Microarchitectural findings in sleep EEG in depression: diagnostic implications. Biological Psychiatry 37, $72-84$.

Armitage, R. \& Hoffmann, R. (1997). Sleep electrophysiology of major depressive disorders. Current Review of Mood and Anxiety Disorders 1, 139-151.

Armitage, R., Hoffmann, R., Loewy, D. \& Moffitt, A. (1989). 
Variations in period-analysed EEG asymmetry in REM and NREM sleep. Psychophysiology 26, 329-336.

Armitage, R., Calhoun, J. S., Rush, A. J. \& Roffwarg, H. P. (1992a). Comparison of the delta EEG in the first and second non-REM periods in depressed adults and normal controls. Psychiatry Research 41, 65-72.

Armitage, R., Roffwarg, H. P., Rush, A. J., Calhoun, J. S., Purdy, D. G. \& Giles, D. E. (1992 b). Digital period analysis of sleep EEG in depression. Biological Psychiatry 31, 52-68.

Armitage, R., Hoffmann, R. \& Moffitt, A. (1992c). Interhemispheric EEG activity in sleep and wakefulness: individual differences in the basic rest-activity cycle (BRAC). In The Neuropsychology of Sleep and Dreaming (ed. J. S. Antrobus and M. Bertini), pp. 17-45. Lawrence Erlbaum \& Associates: Hillsdale.

Armitage, R., Roffwarg, H. P. \& Rush, A. J. (1993). Digital period analysis of EEG in depression: periodicity, coherence, and interhemispheric relationships during sleep. Progress in Neuropsychopharmacology and Biological Psychiatry 17, 363-372.

Armitage, R., Rush, A. J., Trivedi, M., Cain, J. \& Roffwarg, H. P. (1994). The effects of nefazodone on sleep architecture in depression. Neuropsychopharmacology 10, 123-127.

Armitage, R., Hudson, A., Trivedi, M. \& Rush, A. J. (1995). Sex differences in the distribution of EEG frequencies during sleep unipolar depressed outpatients. Journal of Affective Disorders 34, 121-129.

Armitage, R., Hoffmann, R., Trivedi, M. \& Rush, A. J. (1997). Sleep macro- and microarchitecture in depression: age and gender effects. Sleep Research 26, 283

Armitage, R., Hoffmann, R. F., M. \& Rush, A. J. (1999). Temporal characteristics of delta activity during REM and NREM sleep in depressed outpatients and healthy adults: group and sex effects. Sleep (submitted).

Beersma, D. G. M., Daan, S. \& van den Hoofdakker, R. H. (1984) Distribution of REM latencies and other sleep phenomena in depression as explained by a single ultradian rhythm disturbance. Sleep 7, 126-136.

Benca, R. M., Obermeyer, W. H., Thisted, R. A. \& Gillin, J. C. (1992). Sleep and psychiatric disorders. A meta-analysis. Archives of General Psychiatry 49, 651-668.

Borbély, A. A. \& Wirz-Justice, A. (1982). Sleep, sleep deprivation and depression. A hypothesis derived from a model of sleep regulation. Human Neurobiology 1, 205-210.

Borbély, A. A., Tobler, I., Loepfe, M., Kupfer, O. J., Ulrich, R. F., Grochocinski, V., Dorman, J. \& Mathews, G. (1984). All-night spectral analysis of the sleep EEG in untreated depressions and normal controls. Psychiatry Research 12, 27-33.

Broughton, R. (1975). Biorhythmic variations in consciousness and psychological function. Canadian Psychology Review 16, 217-239.

Buysse, D. J., Kupfer, D. J., Frank, E., Monk, T. H. \& Ritenour, A. (1994). Do electro-encephalographic sleep studies predict recurrence in depressed patients successfully treated with psychotherapy? Depression 2, 105-108.

Dew, M. A., Reynolds, C. F., III, Buysse, D. J., Houck, P. R., Hoch, C. C., Monk, T. H. \& Kupfer, D. J. (1996). Electroencephalographic sleep profiles during depression. Effects of episode duration and other clinical and psychosocial factors in older adults. Archives of General Psychiatry, 53, 148-156.

Dijk, D. J., Beersmam, D. G. M. \& Bloem, G. M. (1989). Sex differences in the sleep EEG of young adults: visual scoring and spectral analysis. Sleep 12, 500-507.

Ehlers, C. L. \& Kupfer, D. J. (1997). Slow-wave sleep: do young adult men and women age differently. Journal of Sleep Research $\mathbf{6}$, 211-215.

Geering, B. A., Achermann, P., Eggimann, F. \& Borbély, A. A (1993). Period-amplitude analysis and power spectral analysis: a comparison based on all night sleep EEG recordings. Journal of Sleep Research 2, 121-129.

Gillin, J. C., Sutton, L., Ruiz, C., Kelsoe, J., Dupont, R. M., Darko, D., Risch, S. C., Golshan, S. \& Janowsky, D. (1991). The cholinergic rapid eye movement induction test with arecoline in depression. Archives of General Psychiatry 48, 264-270.
Gottman, J. (1981). Time Series Analysis. Cambridge University Press: Cambridge.

Halberg, F. (1977). President's Letter: Chronobiology in 1977. Chronobiologia 4, 255-263.

Halbreich, U. \& Lumley, L. A. (1993). The multiple interactional biological processes that might lead to depression and gender differences in its appearance. Journal of Affective Disorders 29, 159-173.

Hamilton, M. (1960). A rating scale for depression. Journal of Neurology, Neurosurgery and Psychiatry 23, 56-62.

Healy, D. (1987). Rhythm and blues. Neurochemical, neuropharmacological and neuropsychological implications of hypothesis of circadian rhythm dysfunction in affective disorders. Psychopharmacology 93, 271-285.

Healy, D. \& Waterhouse, J. M. (1995). The circadian system and the therapeutics of the affective disorders. Pharmacology and Therapeutics 65, 241-263.

Heller, W. (1993). Gender differences in depression: perspectives from neuropsychology. Journal of Affective Disorders 29, 129-143.

Hendrickse, W. A., Roffwarg, H. P., Grannemann, B. D., Orsulak, P. J., Armitage, R., Cain, J. W., Battaglia, J., Debus, J. R. \& Rush, A. J. (1994). The effects of fluoxetine on the polysomnogram of depressed outpatients: a pilot study. Neuropsychopharmacology 10, 85-91.

Hoffmann, R. F., Moffitt, A. R., Shearer, J. C., Sussman, P. S. \& Wells, R. B. (1979). Conceptual and methodological considerations towards the development of computer-controlled research on the electro-physiology of sleep. Waking and Sleeping 3, 1-16.

Janowsky, D. S., Halbreigh, U. \& Rausch, J. (1996). Association among ovarian hormones, other hormones, emotional disorders, and neurotransmitters. In Psychopharmacology and Women. Sex, Gender, and Hormones (ed. M. F. Jensvold, U. Halbreigh and J. A. Hamilton), pp. 929-935. American Psychiatric Press, Inc.: Washington, DC.

Jantsch, E. (1980 The Self-Organizing Universe. Scientific and Human Implications of the Emerging Paradigm of Evolution. Pergamon Press: New York.

Kessler, R. C., McGonagle, K. A., Swartz, M., Blazer, D. G. \& Nelson, C. B. (1993). Sex and depression in the National Comorbidity Survey. I: Liftetime prevalence, chronicity and recurrence. Journal of Affective Disorders 29, 85-96.

Kessler, R. C., McGonagle, K. A., Zhao, S., Nelson, C. B., Hughes, M., Eshleman, S., Wittchen, H. U. \& Kendler, K. S. (1994). Lifetime and 12-month prevalence of DSM-III-R psychiatric disorders in the United States. Results from the National Comorbidity Survey. Archives of General Psychiatry 51, 8-19.

Klein, R. \& Armitage, R. (1979). Rhythms in human performance: $1 \frac{1}{2}$-hour oscillations in cognitive style. Science 204, 1326-1328.

Kleitman, N. (1963). Sleep and Wakefulness. University of Chicago Press: Chicago.

Ktonas, P. (1987). Period-amplitude EEG analysis. Sleep 10, 505-507. Ktonas, P. \& Gosalia, A. (1981). Spectral analysis vs. periodamplitude analysis of narrowband EEG activity: a comparison based on the sleep delta frequency band. Sleep 4, 193-206.

Kupfer, D. J., Weiss, B. L., Petre, T. P. \& Foster, F. G. (1974). First night effect revisited: a clinical note. Journal of Nervous and Mental Disease 159, 205-209.

Kupfer, D. J., Ulrich, R. F., Coble, P. A., Jarrett, D. B., Grochocinski, V. J., Doman, J., Matthews, G. \& Bórbely, A. A. (1984). Application of automated REM and slow wave sleep analysis: I. Normal and depressed subjects. Psychiatry Research 13, 325-334.

Kupfer, D. J., Grochocinski, V. J. \& McEachran, A. B. (1986a). Relationship of awakening and delta sleep in depression. Psychiatry Research 19, 297-304.

Kupfer, D. J., Reynolds, C. F., III, Ulrich, R. F. \& Grochocinski, V. J. (1986b). Comparison of automated REM and slow-wave sleep analysis in young and middle-aged depressed subjects. Biological Psychiatry 21, 189-200.

Kupfer, D. J., Reynolds, C. F., III \& Ehlers, C. L. (1989a) Comparison of EEG sleep measures among depressive subtypes and controls in older individuals. Psychiatry Research 27, 13-21. 
Kupfer, D. J., Frank, E. \& Ehlers, C. L. (1989b). EEG sleep in young depressives: first and second night effects. Biological Psychiatry 25, 87-97.

Kupfer, D. J., Frank, E., McEachran, A. B. \& Grochocinski, V. J. (1990). Delta sleep ratio: a biological correlate of early recurrence in unipolar affective disorder. Archives of General Psychiatry 47, 1100-1105.

Manseau, C. \& Broughton, R. (1984). Bilaterally synchronous ultradian EEG rhythms in awake adult humans. Psychophysiology 21, 265-273.

Mendelson, W. B., Sack, D. A., James, S. P., Martin, J. V., Wagner, R., Garnett, D., Milton, J. \& Wehr, T. A. (1987). Frequency analysis of sleep EEG in depression. Psychiatry Research 21, 89-94.

Mistlberger, R. \& Rusak, B. (1989). Mechanisms and models of the circadian timekeeping system. In Principles and Practice of Sleep Medicine (ed. M. H. Kryger, T. Roth and W. C. Dement), pp. 141-152. W. B. Saunders Company: Philadelphia.

Monk, T. H., Buysse, D. J., Frank, E., Kupfer, D. J., Dettling, J. \& Ritenour, A. M. (1994). Nocturnal and circadian body temperatures of depressed outpatients during symptomatic and recovered states. Psychiatry Research 51, 297-311.

Nunez, P. (1981). Electrical Fields of the Brain. Oxford University Press: New York.

Okawa, M., Matousek, M. \& Peterson, I. (1984). Spontaneous vigilance fluctuations in the daytime. Psychophysiology 21, 207-211.

Pedhazur, E. J. (1982). Multiple Regression in Behavioral Research, p. 232ff. Hold, Rinehart \& Winston: New York.

Pigeau, R. A., Hoffmann, R. F. \& Moffitt, A. R. (1981). A multivariate comparison between two EEG analysis techniques: period analysis and fast Fourier transform. Electroencephalography and Clinical Neurophysiology 52, 656-658.

Pollock, V. E. \& Schneider, L. S. (1989). Topographic EEG alpha in recovered depressed elderly. Journal of Abnormal Psychology 98, 268-273.

Rechtschaffen, A. \& Kales, A. (1968). A Manual of Standardized Terminology, Techniques and Scoring System For Sleep Stages of Human Subjects. National Institute of Health Publication No. 204. US Government Printing Office: Washington, DC
Reynolds, C. F., III \& Kupfer, D. J. (1987). Sleep research in affective illness: state of the art circa 1987. Sleep 10, 199-215.

Reynolds, C. F., III, Kupfer, D. J., Thase, M. E., Frank, E., Jarrett, D. B., Coble, P. A., Hoch, C. C., Buysse, D. J., Simons, A. D. \& Houck, P. R. (1990). Sleep, gender, and depression: an analysis of gender effects on the electroencephalographic sleep of 302 depressed outpatients. Biological Psychiatry 28, 673-684.

Riemann, D. \& Berger, M. (1992). Sleep, age, depression and the cholingeric REM induction test with RS86. Progress in Neuropsychopharmacology and Biological Psychiatry 16, 311-316.

Riemann, D., Hohagen, F., Fleckenstein, P., Schredl, M. \& Berger, M. (1991). The cholinergic REM induction test with RS86 after scopolamine pretreatment in healthy subjects. Psychiatry Research 38, 247-260.

Spitzer, R. L., Williams, J. B. W. \& Gibbons, M. (1986). The Structured Clinical Interview for DMS-III-R (SCID). New York State Psychiatric Research Institute: New York.

Teicher, M. H., Glod, C. A., Harper, D., Magnus, E., Brasher, C., Wren, F. \& Pahlavan, K. (1993). Locomotor activity in depressed children and adolescents: I. Circadian dysregulation. Journal of the American Academy of Child and Adolescent Psychiatry 32, 760-769.

Teicher, M. H., Glod, C. A., Magnus, E., Harper, D., Benson, G., Krueger, K. \& McGreenery, C. E. (1997). Circadian rest-activity disturbances in seasonal affective disorder. Archives of General Psychiatry 54, 124-130.

Trivedi, M. H., Rush, A. J., Armitage, R., Gullion, C. M., Grannemann, B. D. Orsulak, P. J. \& Roffwarg, H. P. (1998). Effects of fluoxetine on the polysomnogram in outpatients with major depression. Neuropsychopharmacology 20, 447-459.

Van den Hoofdakker, R. H. \& Beersma, D. G. (1986). On the contribution of sleep-wake physiology to the explanation and treatment of depression. Psychiatry Research 16, 155-163.

Vogel, G. W., Vogel, F., McAbee, R. S. \& Thurmond, A. J. (1980) Improvement of depression by REM sleep deprivation: new findings and a theory. Archives of General Psychiatry 37, 247-253.

Webb, W. B. (1982). Biological Rhythms, Sleep, and Performance. Wiley \& Sons: New York.

Wirz-Justice, A. (1995). Biological rhythms in mood disorders. In Psychopharmacology: The Fourth Generation of Progress (ed. F. E. Bloom and D. J. Kupfer), pp. 999-1017. Raven Press: New York. 